International Journal of Pure and Applied Mathematics

Volume 90 No. 3 2014, 335-344

ISSN: $1311-8080$ (printed version); ISSN: 1314-3395 (on-line version)

url: http://www.ijpam.eu

doi: http://dx.doi.org/10.12732/ijpam.v90i3.7

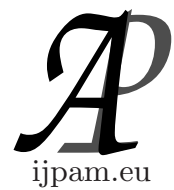

\title{
THE PERIOD MODULO PRODUCT OF CONSECUTIVE FIBONACCI NUMBERS
}

\author{
Narissara Khaochim ${ }^{1}$, Prapanpong Pongsriiam ${ }^{2} \S$ \\ 1,2 Department of Mathematics, Faculty of Science \\ Silpakorn University \\ Ratchamankanai Rd, Nakornpathom, 73000, THAILAND
}

Abstract: Let $F_{n}$ be the $n$th Fibonacci number. The period modulo $m$, denoted by $s(m)$, is the smallest positive integer $k$ for which $F_{n+k} \equiv F_{n}(\bmod m)$ for all $n \geq 0$. In this paper, we find the period modulo product of consecutive Fibonacci numbers. For instance, we prove that, for $n \geq 1$,

$$
s\left(F_{n} F_{n+1} F_{n+2} F_{n+3}\right)= \begin{cases}n(n+1)(n+2)(n+3), & \text { if } n \neq 0 \quad(\bmod 3), \\ \frac{2 n(n+1)(n+2)(n+3)}{3}, & \text { if } n \equiv 0,9 \quad(\bmod 12), \\ \frac{n(n+1)(n+2)(n+3)}{3}, & \text { if } n \equiv 3,6 \quad(\bmod 12) .\end{cases}
$$

AMS Subject Classification: 11B39

Key Words: Fibonacci sequence, divisibility, Fibonacci entry point, the period modulo $m$

\section{Introduction}

Let $\left(F_{n}\right)_{n \geq 0}$ be the Fibonacci sequence given by $F_{n+2}=F_{n+1}+F_{n}$, for $n \geq 0$, where $F_{0}=0$ and $F_{1}=1$. These number are famous for possessing wonderful properties, see $[2,3,5,6,15]$ for additional references and history. In particular, we will be concerned with the divisibility properties and the periodic nature of the Fibonacci sequence.

Received: September 5, 2013

(C) 2014 Academic Publications, Ltd.

${ }^{\S}$ Correspondence author url: www.acadpubl.eu 
Let $m$ be a positive integer. The order of appearance of $m$ in the Fibonacci sequence, denoted by $z(m)$, is defined as the smallest positive integer $k$ such that $m \mid F_{k}$ (some authors also call it the order of apparition, the rank of apparition, or Fibonacci entry point). The period modulo $m$ of the Fibonacci sequence, denoted by $s(m)$, is defined as the smallest positive integer $k$ such that $F_{n+k} \equiv F_{n}(\bmod m)$ for all $n \geq 0$. There are several results about $z(m)$ and $s(m)$ in the literature. For instance, Stanley [14] shows that if $m$ is an integer greater than 3 then $s\left(F_{m}\right)=2 m$ if $m$ is even and $s\left(F_{m}\right)=4 m$ if $m$ is odd. It is also well known that $z\left(F_{m}\right)=m$ for $m \geq 3$ (see for example [4, p. 221]). For other classical results on $z(m)$ and $s(m)$, we refer the reader to $[1,4,6,12,13,14,15,16,17]$.

Recently, D. Marques $[7,8,9,10,11]$ has obtained a formula of $z(m)$ for various special numbers $m$. Particularly, he obtains the following [10] in 2012.

Theorem 1. (Marques [10])

(i) For $n \geq 3$,

$$
z\left(F_{n} F_{n+1}\right)=n(n+1) .
$$

(ii) For $n \geq 2$,

$$
z\left(F_{n} F_{n+1} F_{n+2}\right)=\left\{\begin{array}{lll}
n(n+1)(n+2), & \text { if } n \equiv 1 \quad(\bmod 2), \\
\frac{n(n+1)(n+2)}{2}, & \text { if } n \equiv 0 \quad(\bmod 2) .
\end{array}\right.
$$

(iii) For $n \geq 1$,

$$
z\left(F_{n} F_{n+1} F_{n+2} F_{n+3}\right)= \begin{cases}\frac{n(n+1)(n+2)(n+3)}{2}, & \text { if } n \neq 0 \quad(\bmod 3), \\ \frac{n(n+1)(n+2)(n+3)}{3}, & \text { if } n \equiv 0,9 \quad(\bmod 12), \\ \frac{n(n+1)(n+2)(n+3)}{6}, & \text { if } n \equiv 3,6 \quad(\bmod 12) .\end{cases}
$$

The above theorem motivates us to study the period $s(n)$ when $n$ is the product of consecutive Fibonacci numbers. Our main results are the formulas for $s\left(F_{n} F_{n+1}\right), s\left(F_{n} F_{n+1} F_{n+2}\right)$, and $s\left(F_{n} F_{n+1} F_{n+2} F_{n+3}\right)$. This task is a bit more difficult than the calculation of, for example, $z\left(F_{n} F_{n+1} F_{n+2} F_{n+3}\right)$ in Theorem 1. This is because the latter only requires the smallest $k$ such that $F_{k} \equiv 0\left(\bmod F_{n} F_{n+1} F_{n+2} F_{n+3}\right)$ while our task is to find the smallest $k$ such that $F_{k} \equiv 0\left(\bmod F_{n} F_{n+1} F_{n+2} F_{n+3}\right)$ and $F_{k+1} \equiv 1\left(\bmod F_{n} F_{n+1} F_{n+2} F_{n+3}\right)$ (see Lemma 2). But with the aid of Lemma 3, our task become easy.

We will give some lemmas in the next section. Then we will give the main results in the last section. 


\section{Preliminaries}

We recall some facts on Fibonacci numbers for the convenience of the reader.

Let $n$ and $m$ be positive integers. The following results are well known and will be used throughout this article:

$$
\text { For } n \geq 3, F_{n} \mid F_{m} \text { if and only if } n \mid m \text {. }
$$

For $n \geq 1, F_{n-1} F_{n+1}-F_{n}^{2}=(-1)^{n}$ (The Cassini's formula).

For $k, n \geq 1$ and $r \geq 0, F_{k n+r}=\sum_{j=0}^{k}\left(\begin{array}{c}k \\ j\end{array}\right) F_{n}^{j} F_{n-1}^{k-j} F_{j+r}$

For $n \geq 1, F_{n+1}^{2} \equiv F_{n-1}^{2} \equiv(-1)^{n} \quad\left(\bmod F_{n}\right)$.

Identity (1) and (2) can be found, for example, in [3, 5, 6, 15]. Identity (3) and (4) might be less well known, so we will give a proof here for completeness.

Let $\alpha=\frac{1+\sqrt{5}}{2}$ and $\beta=\frac{1-\sqrt{5}}{2}$. It is well known that $F_{n}=\frac{\alpha^{\mathrm{n}}-\beta^{\mathrm{n}}}{\alpha-\beta}$. By solving the equation, $\alpha^{n}-\beta^{n}=(\alpha-\beta) F_{n}$, and $\alpha \cdot \alpha^{n}-\beta \cdot \beta^{n}=(\alpha-\beta) F_{n+1}$, for $\alpha^{n}$ and $\beta^{n}$, we obtain $\alpha^{n}=\alpha F_{n}+F_{n-1}, \beta^{n}=\beta F_{n}+F_{n-1}$. Let $k, n \geq 1$ and $r \geq 0$. Then

$$
\begin{aligned}
F_{k n+r} & =\frac{\alpha^{k n+r}-\beta^{k n+r}}{\alpha-\beta} \\
& =\frac{1}{\alpha-\beta}\left[\left(\alpha F_{n}+F_{n-1}\right)^{k} \alpha^{r}-\left(\beta F_{n}+F_{n-1}\right)^{k} \beta^{r}\right] \\
& =\frac{1}{\alpha-\beta}\left[\sum_{j=0}^{k}\left(\begin{array}{c}
k \\
j
\end{array}\right)\left(\alpha F_{n}\right)^{j} F_{n-1}^{k-j} \alpha^{r}-\sum_{j=0}^{k}\left(\begin{array}{c}
k \\
j
\end{array}\right)\left(\beta F_{n}\right)^{j} F_{n-1}^{k-j} \beta^{r}\right. \\
& =\frac{1}{\alpha-\beta} \sum_{j=0}^{k}\left[\left(\begin{array}{c}
k \\
j
\end{array}\right) F_{n}^{j} F_{n-1}^{k-j}\left(\alpha^{j+r}-\beta^{j+r}\right)\right] \\
& =\sum_{j=0}^{k}\left(\begin{array}{c}
k \\
j
\end{array}\right) F_{n}^{j} F_{n-1}^{k-j} F_{j+r} .
\end{aligned}
$$

This proves (3). Next we prove (4). Let $n \geq 1$. By the Cassini's formula, we have $F_{n} F_{n+2}-F_{n+1}^{2}=(-1)^{n+1}$. So $F_{n+1}^{2}=F_{n} F_{n+2}-(-1)^{n+1} \equiv(-1)^{n+2} \equiv$ $(-1)^{n}\left(\bmod F_{n}\right)$ and $F_{n-1}^{2}=\left(F_{n+1}-F_{n}\right)^{2} \equiv F_{n+1}^{2} \equiv(-1)^{n}\left(\bmod F_{n}\right)$. This proves (4).

The next result is actually an equivalent definition for the period of the Fibonacci sequence modulo $m$. It appeared for example in $[12,16,17]$. 
Lemma 2. Let $m$ be a positive integer. Then $s(m)$ is equal to the smallest positive integer $k$ such that $F_{k} \equiv 0(\bmod m)$ and $F_{k+1} \equiv 1(\bmod m)$.

Proof. Let $m$ be a positive integer and let $k$ be the smallest positive integer such that $F_{k} \equiv 0(\bmod m)$ and $F_{k+1} \equiv 1(\bmod m)$. We will prove that $F_{n+k} \equiv$ $F_{n}(\bmod m)$ for all $n \geq 0$ by strong induction on $n$. It is clearly true for $n=0$ and $n=1$. Assume that it is valid for any integer $j$ such that $0 \leq j \leq n$. Thus

$$
F_{(n+1)+k}=F_{n+k+1}=F_{n+k}+F_{n+k-1} \equiv F_{n}+F_{n-1} \equiv F_{n+1} \quad(\bmod m) .
$$

Therefore $F_{n+k} \equiv F_{n}(\bmod m)$ for all $n \geq 0$. Suppose that $r$ is a positive integer such that $F_{n+r} \equiv F_{n}(\bmod m)$ for all $n \geq 0$. Then $F_{r} \equiv F_{0} \equiv 0$ $(\bmod m)$ and $F_{r+1} \equiv F_{1} \equiv 1(\bmod m)$. Thus $k \leq r$. Therefore $k$ is the smallest positive integer such that $F_{n+k} \equiv F_{n}(\bmod m)$ for all $n \geq 0$. We conclude that $s(m)=k$.

It is shown in [4] that $z(n) \mid s(n)$ for every $n \geq 1$. So it is natural to define the quantity $t(n)=\frac{s(n)}{z(n)}$. So $t(n)$ is an integer for all $n \geq 1$. A useful result on $t(n)$ is obtained by Vinson[16] as follows.

Lemma 3. (Vinson [16]) The following statement holds.

(i) $t(m)=4$ if $m>2$ and $z(m)$ is odd,

(ii) $t(m)=1$ if $8 \nmid m$ and $2 \mid z(p)$ but $4 \nmid z(p)$ for every odd prime $p$ dividing $m$, and

(iii) $t(m)=2$ for other $m$.

\section{Main Results}

In this section, we give the proof of the main theorems. As mentioned before, Lemma 2 gives an equivalent definition of $s(n)$ and we will use Lemma 2 without further referring.

Theorem 4. $s\left(F_{1} F_{2}\right)=s(1)=1, s\left(F_{2} F_{3}\right)=s(2)=3$, and $s\left(F_{n} F_{n+1}\right)=$ $2 n(n+1)$ for every $n \geq 3$.

Proof. It is straightforward to verify that $s\left(F_{1} F_{2}\right)=s(1)=1, s\left(F_{2} F_{3}\right)=$ $s(2)=3$. So we let $n \geq 3$. By Theorem 1 , we have $z\left(F_{n} F_{n+1}\right)=n(n+1)$, which is even. Then by Lemma 3 , we see that $t\left(F_{n} F_{n+1}\right)=1$ or 2 . Therefore

$$
s\left(F_{n} F_{n+1}\right)=n(n+1) \text { or } s\left(F_{n} F_{n+1}\right)=2 n(n+1) .
$$


First we consider $F_{n(n+1)+1}\left(\bmod F_{n}\right)$. By $(3)$, we have

$$
F_{n(n+1)+1}=\sum_{j=0}^{n+1}\left(\begin{array}{c}
n+1 \\
j
\end{array}\right) F_{n}^{j} F_{n-1}^{n+1-j} F_{j+1} \equiv F_{n-1}^{n+1} \quad\left(\bmod F_{n}\right) .
$$

We consider the following two cases:

Case 1: If $n$ is even, then by (4), we have $F_{n-1}^{n+1} \equiv\left(F_{n-1}^{2}\right)^{\frac{\mathrm{n}}{2}} F_{n-1} \equiv$ $(-1)^{n\left(\frac{\mathrm{n}}{2}\right)} F_{n-1} \equiv F_{n-1} \not \equiv 1\left(\bmod F_{n}\right)$. This is because $2 \leq F_{n-1}<F_{n}$.

Case 2: Assume that $n$ is odd. Then by $(4), F_{n-1}^{n+1} \equiv\left(F_{n-1}^{2}\right)^{\frac{n+1}{2}} \equiv$ $(-1)^{n\left(\frac{\mathrm{n}+1}{2}\right)} \equiv(-1)^{\frac{\mathrm{n}+1}{2}}\left(\bmod F_{n}\right)$. Since $(-1)^{\frac{\mathrm{n}+1}{2}}=1$ if and only if $4 \mid n+1$, we see that $F_{n-1}^{n+1} \equiv 1\left(\bmod F_{n}\right)$ if and only if $n \equiv 3(\bmod 4)$. We conclude that

$$
F_{n(n+1)+1} \equiv 1 \quad\left(\bmod F_{n}\right) \text { if and only if } n \equiv 3 \quad(\bmod 4) .
$$

Next we consider $F_{n(n+1)+1}\left(\bmod F_{n+1}\right)$. By $(3)$, we have

$$
F_{n(n+1)+1}=\sum_{j=0}^{n}\left(\begin{array}{l}
n \\
j
\end{array}\right) F_{n+1}^{j} F_{n}^{n-j} F_{j+1} \equiv F_{n}^{n} \quad\left(\bmod F_{n+1}\right) .
$$

Similar to the proof of (6), we apply (4) to obtain the following:

If $n$ is odd, then $F_{n}^{n} \equiv\left(F_{n}^{2}\right)^{\frac{\mathrm{n}-1}{2}} F_{n} \equiv(-1)^{(n+1)\left(\frac{\mathrm{n}-1}{2}\right)} F_{n} \equiv F_{n} \not \equiv 1\left(\bmod F_{n+1}\right)$.

If $n$ is even, then $F_{n}^{n} \equiv\left(F_{n}^{2}\right)^{\frac{\mathrm{n}}{2}} \equiv(-1)^{(n+1)\left(\frac{\mathrm{n}}{2}\right)} \equiv(-1)^{\frac{\mathrm{n}}{2}}\left(\bmod F_{n+1}\right)$, which is congruent to $1\left(\bmod F_{n+1}\right)$ if and only if $4 \mid n$. Therefore we obtain

$$
F_{n(n+1)+1} \equiv 1 \quad\left(\bmod F_{n+1}\right) \text { if and only if } n \equiv 0 \quad(\bmod 4) .
$$

Since $\operatorname{gcd}\left(F_{n}, F_{n+1}\right)=1$, we have

$$
\begin{aligned}
F_{k} \equiv 1 \quad\left(\bmod F_{n} F_{n+1}\right) \text { if and only if } F_{k} & \equiv 1 & \left(\bmod F_{n}\right) \text { and } \\
F_{k} & \equiv 1 & \left(\bmod F_{n+1}\right) .
\end{aligned}
$$

From (6), (7) and (8), we see that there is no $n \in \mathbb{N}$ satisfying $F_{n(n+1)+1} \equiv 1$ $\left(\bmod F_{n} F_{n+1}\right)$. Hence $s\left(F_{n} F_{n+1}\right) \neq n(n+1)$. Now by $(5)$, we can conclude that $s\left(F_{n} F_{n+1}\right)=2 n(n+1)$. This completes the proof.

Remark 1. We can also directly calculate $F_{2 n(n+1)+1}\left(\bmod F_{n} F_{n+1}\right)$ by applying Identity (3) and (4) as follows.

$$
F_{2 n(n+1)+1}=\sum_{j=0}^{2(n+1)}\left(\begin{array}{c}
2(n+1) \\
j
\end{array}\right) F_{n}^{j} F_{n-1}^{2(n+1)-j} F_{j+1}
$$




$$
\begin{aligned}
& \equiv F_{n-1}^{2(n+1)} \equiv\left(F_{n-1}^{2}\right)^{n+1} \equiv(-1)^{n(n+1)} \equiv 1 \quad\left(\bmod F_{n}\right) \\
F_{2 n(n+1)+1} & =\sum_{j=0}^{2 n}\left(\begin{array}{c}
2 n \\
j
\end{array}\right) F_{n+1}^{j} F_{n}^{2 n-j} F_{j+1} \equiv F_{n}^{2 n} \equiv\left(F_{n}^{2}\right)^{n} \\
& \equiv(-1)^{(n+1) n} \equiv 1 \quad\left(\bmod F_{n+1}\right) .
\end{aligned}
$$

Since $\operatorname{gcd}\left(F_{n}, F_{n+1}\right)=1$, we have $F_{2 n(n+1)+1} \equiv 1\left(\bmod F_{n} F_{n+1}\right)$, as required.

Note. also that, from this point on, we will apply (4) without referring to it.

Theorem 5. $s\left(F_{1} F_{2} F_{3}\right)=s(2)=3$ and for $n \geq 2$, we have

$$
s\left(F_{n} F_{n+1} F_{n+2}\right)=\left\{\begin{array}{lll}
2 n(n+1)(n+2), & \text { if } n \equiv 1 \quad(\bmod 2), \\
n(n+1)(n+2), & \text { if } n \equiv 0 \quad(\bmod 2) .
\end{array}\right.
$$

Proof. It easy to verify that $s\left(F_{1} F_{2} F_{3}\right)=s(2)=3$. So we let $n \geq 2$. We split the proof into two cases:

Case 1: Assume that $n$ is even. Then $n+2$ is even and $2 \mid \frac{n(n+1)(n+2)}{2}$. Therefore, by Theorem $1, z\left(F_{n} F_{n+1} F_{n+2}\right)$ is even. So, by Lemma $3, t\left(F_{n} F_{n+1}\right.$ $\left.F_{n+2}\right)=1$ or 2 . We will show that $t\left(F_{n} F_{n+1} F_{n+2}\right)=2$. By Lemma 3, it suffices to find a prime $p \mid F_{n} F_{n+1} F_{n+2}$ such that $4 \mid z(p)$. Since $n$ is even, $4 \mid n$ or $4 \mid n+2$. Then, by (1), $F_{4} \mid F_{n}$ or $F_{4} \mid F_{n+2}$ and therefore $F_{4} \mid F_{n} F_{n+1} F_{n+2}$. That is $3 \mid F_{n} F_{n+1} F_{n+2}$. Since $z(3)=4$, we have $t\left(F_{n} F_{n+1} F_{n+2}\right)=2$, as desired. In conclusion, if $n$ is even

$$
s\left(F_{n} F_{n+1} F_{n+2}\right)=2 z\left(F_{n} F_{n+1} F_{n+2}\right)=n(n+1)(n+2) .
$$

Case 2: Assume that $n$ is odd. By Theorem 1, $z\left(F_{n} F_{n+1} F_{n+2}\right)=n(n+1)(n+2)$ which is an even number. So, by Lemma 3 , we obtain $t\left(F_{n} F_{n+1} F_{n+2}\right)=1$ or 2 . Thus

$$
s\left(F_{n} F_{n+1} F_{n+2}\right)=n(n+1)(n+2) \text { or } s\left(F_{n} F_{n+1} F_{n+2}\right)=2 n(n+1)(n+2) .
$$

We consider $F_{n(n+1)(n+2)+1}\left(\bmod F_{n+1}\right)$. By $(3)$, we have

$$
F_{n(n+1)(n+2)+1}=\sum_{j=0}^{n(n+2)}\left(\begin{array}{c}
n(n+2) \\
j
\end{array}\right) F_{n+1}^{j} F_{n}^{n(n+2)-j} F_{j+1} \equiv F_{n}^{n(n+2)} \quad\left(\bmod F_{n+1}\right) .
$$


Since $n$ is odd, $n(n+2)$ is odd. So, by (4), we have

$$
F_{n}^{n(n+2)} \equiv\left(F_{n}^{2}\right)^{\frac{\mathrm{n}(\mathrm{n}+2)-1}{2}} F_{n} \equiv(-1)^{(n+1) \frac{\mathrm{n}(\mathrm{n}+2)-1}{2}} F_{n} \equiv F_{n} \not \equiv 1 \quad\left(\bmod F_{n+1}\right) .
$$

Thus $F_{n(n+1)(n+2)+1} \not \equiv 1\left(\bmod F_{n} F_{n+1} F_{n+2}\right)$. Therefore

$$
s\left(F_{n} F_{n+1} F_{n+2}\right) \neq n(n+1)(n+2) .
$$

Now by (9), we conclude that $s\left(F_{n} F_{n+1} F_{n+2}\right)=2 n(n+1)(n+2)$. This completes the proof.

Remark 2. We can also calculate $F_{2 n(n+1)(n+2)+1}\left(\bmod F_{n} F_{n+1} F_{n+2}\right)$ by applying (3) as follows

$$
\begin{aligned}
& F_{2 n(n+1)(n+2)+1}=\sum_{j=0}^{2(n+1)(n+2)}\left(\begin{array}{c}
2(n+1)(n+2) \\
j
\end{array}\right) F_{n}^{j} F_{n-1}^{2(n+1)(n+2)-j} F_{j+1} \\
& \equiv F_{n-1}^{2(n+1)(n+2)} \equiv\left(F_{n-1}^{2}\right)^{(n+1)(n+2)} \equiv(-1)^{n(n+1)(n+2)} \equiv 1 \quad\left(\bmod F_{n}\right) . \\
& F_{2 n(n+1)(n+2)+1}=\sum_{j=0}^{2 n(n+2)}\left(\begin{array}{c}
2 n(n+2) \\
j
\end{array}\right) F_{n+1}^{j} F_{n}^{2 n(n+2)-j} F_{j+1} \\
& \equiv F_{n}^{2 n(n+2)} \equiv\left(F_{n}^{2}\right)^{n(n+2)} \equiv(-1)^{(n+1)(n)(n+2)} \equiv 1 \quad\left(\bmod F_{n+1}\right) . \\
& F_{2 n(n+1)(n+2)+1}=\sum_{j=0}^{2 n(n+1)}\left(\begin{array}{c}
2 n(n+1) \\
j
\end{array}\right) F_{n+2}^{j} F_{n+1}^{2 n(n+1)-j} F_{j+1} \\
& \equiv F_{n+1}^{2 n(n+1)} \equiv\left(F_{n+1}^{2}\right)^{n(n+1)} \equiv(-1)^{(n+2)(n)(n+1)} \equiv 1 \quad\left(\bmod F_{n+2}\right) .
\end{aligned}
$$

Since the number $F_{n}, F_{n+1}, F_{n+2}$ are pairwise relatively prime, we have

$$
F_{2 n(n+1)(n+2)+1} \equiv 1 \quad\left(\bmod F_{n} F_{n+1} F_{n+2}\right),
$$

as required.

Theorem 6. For $n \geq 1$,

$$
s\left(F_{n} F_{n+1} F_{n+2} F_{n+3}\right)= \begin{cases}n(n+1)(n+2)(n+3), & \text { if } n \neq \equiv 0 \quad(\bmod 3), \\ \frac{2 n(n+1)(n+2)(n+3)}{3}, & \text { if } n \equiv 0,9 \quad(\bmod 12), \\ \frac{n(n+1)(n+2)(n+3)}{3}, & \text { if } n \equiv 3,6 \quad(\bmod 12) .\end{cases}
$$


Proof. Let $n \geq 3$. We split the proof into three cases:

Case 1: Assume that $n \not \equiv 0(\bmod 3)$. By Theorem $1, z\left(F_{n} F_{n+1} F_{n+2} F_{n+3}\right)$ $=\frac{n(n+1)(n+2)(n+3)}{2}$. Since $4|n(n+1)(n+2)(n+3), 2| \frac{n(n+1)(n+2)(n+3)}{2}$. So, by Lemma 3 , we have $t\left(F_{n} F_{n+1} F_{n+2} F_{n+3}\right)=1$ or 2 . We will show that $t\left(F_{n} F_{n+1} F_{n+2} F_{n+3}\right)=2$. By Lemma 3 , it suffices to find a prime $p \mid F_{n} F_{n+1} F_{n+2}$ $F_{n+3}$ such that $4 \mid z(p)$, or to show that $8 \mid F_{n} F_{n+1} F_{n+2} F_{n+3}$. Since $n \not \equiv 0$ $(\bmod 3)$, we have $n \equiv 1,2,4,5(\bmod 6)$.

(i) Assume that $n \equiv 4,5(\bmod 6)$. Then $6 \mid n+2$ or $6 \mid n+1$. By (1), we have $F_{6} \mid F_{n+2}$ or $F_{6} \mid F_{n+1}$ and therefore $F_{6} \mid F_{n} F_{n+1} F_{n+2} F_{n+3}$. That is $8 \mid F_{n} F_{n+1} F_{n+2} F_{n+3}$, as desired.

(ii) Assume that $n \equiv 1,2(\bmod 6)$. Then $n \equiv 1,2,7,8(\bmod 12)$. So $4 \mid$ $n+k$ for some $k \in\{0,1,2,3\}$. By (1), we have $F_{4} \mid F_{n+k}$ for some $k \in\{0,1,2,3\}$. That is $3 \mid F_{n+k}$ for some $k \in\{0,1,2,3\}$ and so $3 \mid$ $F_{n} F_{n+1} F_{n+2} F_{n+3}$. Thus 3 is an odd prime dividing $F_{n} F_{n+1} F_{n+2} F_{n+3}$ and $4 \mid z(3)$, as required.

Therefore $t\left(F_{n} F_{n+1} F_{n+2} F_{n+3}\right)=2$. We conclude that

$$
s\left(F_{n} F_{n+1} F_{n+2} F_{n+3}\right)=2 z\left(F_{n} F_{n+1} F_{n+2} F_{n+3}\right)=n(n+1)(n+2)(n+3) .
$$

Case 2: Assume that $n \equiv 0,9(\bmod 12)$. By Theorem $1, z\left(F_{n} F_{n+1} F_{n+2}\right.$ $\left.F_{n+3}\right)=\frac{n(n+1)(n+2)(n+3)}{3}$. Since $n \equiv 0,9(\bmod 12)$, we have $6 \mid n$ or $6 \mid n+3$. So $2 \mid \frac{n(n+1)(n+2)(n+3)}{3}$. Therefore $z\left(F_{n} F_{n+1} F_{n+2} F_{n+3}\right)$ is even. By Lemma 3, we have $t\left(F_{n} F_{n+1} F_{n+2} F_{n+3}\right)=1$ or 2 . We will show that $t\left(F_{n} F_{n+1} F_{n+2} F_{n+3}\right)=2$. By Lemma 3, it suffices to show that $8 \mid F_{n} F_{n+1} F_{n+2} F_{n+3}$. Since $6 \mid n$ or $6 \mid n+3$, we have $F_{6} \mid F_{n}$ or $F_{6} \mid F_{n+3}$. So $F_{6} \mid F_{n} F_{n+1} F_{n+2} F_{n+3}$. That is $8 \mid F_{n} F_{n+1} F_{n+2} F_{n+3}$, as desired. Therefore $t\left(F_{n} F_{n+1} F_{n+2} F_{n+3}\right)=2$ and hence

$$
s\left(F_{n} F_{n+1} F_{n+2} F_{n+3}\right)=2 z\left(F_{n} F_{n+1} F_{n+2} F_{n+3}\right)=\frac{2 n(n+1)(n+2)(n+3)}{3} .
$$

Case 3: Assume that $n \equiv 3,6(\bmod 12)$. Then, by Theorem 1 , we have

$$
z\left(F_{n} F_{n+1} F_{n+2} F_{n+3}\right)=\frac{n(n+1)(n+2)(n+3)}{6} .
$$

We will show that $t\left(F_{n} F_{n+1} F_{n+2} F_{n+3}\right)=2$. By Lemma 3 , it suffices to show that

$$
z\left(F_{n} F_{n+1} F_{n+2} F_{n+3}\right) \text { is even and } 8 \mid F_{n} F_{n+1} F_{n+2} F_{n+3} \text {. }
$$


(i) Assume that $n \equiv 3(\bmod 12)$. Then $6 \mid n+3$ and $2 \mid n+1$. So $2 \mid$ $\frac{n(n+1)(n+2)(n+3)}{6}$. Thus $z\left(F_{n} F_{n+1} F_{n+2} F_{n+3}\right)$ is even. Since $6 \mid n+3$, $F_{6} \mid F_{n+3}$. So $F_{6} \mid F_{n} F_{n+1} F_{n+2} F_{n+3}$. That is $8 \mid F_{n} F_{n+1} F_{n+2} F_{n+3}$.

(ii) Assume that $n \equiv 6(\bmod 12)$. Then $6 \mid n$ and $2 \mid n+2$. So $2 \mid$ $\frac{n(n+1)(n+2)(n+3)}{6}$. Thus $z\left(F_{n} F_{n+1} F_{n+2} F_{n+3}\right)$ is even. Since $6\left|n, F_{6}\right| F_{n}$. So $F_{6} \mid F_{n} F_{n+1} F_{n+2} F_{n+3}$. That is $8 \mid F_{n} F_{n+1} F_{n+2} F_{n+3}$.

In any case, $z\left(F_{n} F_{n+1} F_{n+2} F_{n+3}\right)$ is even and $8 \mid F_{n} F_{n+1} F_{n+2} F_{n+3}$. Therefore

$$
t\left(F_{n} F_{n+1} F_{n+2} F_{n+3}\right)=2 .
$$

We conclude that

$$
s\left(F_{n} F_{n+1} F_{n+2} F_{n+3}\right)=2 z\left(F_{n} F_{n+1} F_{n+2} F_{n+3}\right)=\frac{n(n+1)(n+2)(n+3)}{3} .
$$

This completes the proof.

\section{Acknowledgments}

The first author receives scholarship from DPST Thailand. The second author receives financial support from Faculty of Science, Silpakorn University, Thailand, contract number RGP 2555-07.

\section{References}

[1] A. Andreassian, Fibonacci sequences modulo $m$, The Fibonacci Quarterly, 12, No.1 (1974), 51-65.

[2] A. Benjamin and J. Quinn, The Fibonacci numbers-exposed more discretely, Math. Mag., 76, No.3 (2013), 182-192.

[3] R. L. Graham, D. E. Knuth and O. Patashnik, Concrete Mathematics, Second edition, Addison-Wesley, USA (1994).

[4] J. H. Halton, On the divisibility properties of Fibonacci numbers, The Fibonacci Quarterly, 4, No.3 (1996), 217-240.

[5] D. Kalman, and R. Mena, The Fibonacci numbers-exposed, Math. Mag., 76, No.3 (2003), 167-181. 
[6] T. Koshy, Fibonacci and Lucas Numbers with Applications, Wiley, New York (2001).

[7] D. Marques, The order of appearance of the product of consecutive Lucas numbers, The Fibonacci Quarterly, 51, No.1 (2013), 38-43.

[8] D. Marques, Fixed points of the order of appearance in the Fibonacci sequence, The Fibonacci Quarterly, 50, No.4 (2012), 346-351.

[9] D. Marques, The order of appearance of powers of Fibonacci and Lucas numbers, The Fibonacci Quarterly, 50, No.3 (2012), 239-245.

[10] D. Marques, The order of appearance of product of consecutive Fibonacci numbers, The Fibonacci Quarterly, 50, No.2 (2012), 132-139.

[11] D. Marques, On the order of appearance of integers at most one away from Fibonacci numbers, The Fibonacci Quarterly, 50, No.1 (2012), 36-43.

[12] D. W. Robinson, The Fibonacci matrix modulo m, The Fibonacci Quarterly, 1, No.2 (1963), 29-35.

[13] T. E. Stanley, Some remarks on the periodicity of the Sequence of Fibonacci Numbers, The Fibonacci Quarterly, 14, No.1 (1976), 52-54.

[14] T. E. Stanley, A note on the Sequence of Fibonacci Numbers, Math. Mag., 44, No.1 (1971), 19-22.

[15] S. Vajda, Fibonacci and Lucas Numbers and the Golden Section: Theory and Applications, Dover Publications, New York (2008).

[16] J. Vinson, The relation of the period modulo $m$ to the rank of apparition of $m$ in the Fibonacci sequence, The Fibonacci Quarterly, 1, No.2 (1963), $37-45$.

[17] D. D. Wall, Fibonacci series modulo m, Amer. Math. Monthly, 67, No.6 (1960), 525-532. 